\title{
Predicting Subset Sum Pseudorandom Generators
}

\author{
Joachim von zur Gathen ${ }^{1}$ and Igor E. Shparlinski ${ }^{2}$ \\ 1 Fakultät für Elektrotechnik, Informatik und Mathematik, \\ Universität Paderborn, \\ 33095 Paderborn, Germany \\ gathen@upb.de \\ http://www-math.upb.de/ ${ }^{\sim}$ aggathen \\ 2 Department of Computing, Macquarie University, \\ NSW 2109, Australia \\ igor@comp.mq.edu.au \\ http://www. comp.mq.edu.au/ igor
}

\begin{abstract}
We consider the subset sum pseudorandom generator, introduced by Rueppel and Massey in 1985 and given by a linearly recurrent bit sequence $u_{0}, u_{1}, \ldots$ of order $n$ over $\mathbb{Z}_{2}$, and weights $w=$ $\left(w_{0}, \ldots, w_{n-1}\right) \in R^{n}$ for some ring $R$. The rings $R=\mathbb{Z}_{m}$ are of particular interest. The $i$ th value produced by this generator is $\sum_{0<j<n} u_{i+j} w_{j}$. It is also recommended to discard about $\log n$ least significant bits of the result before using this sequence. We present several attacks on this generator (with and without the truncation), some of which are rigorously proven while others are heuristic. They work when one "half" of the secret is given, either the control sequence $u_{j}$ or the weights $w_{j}$. Our attacks do not mean that the generator is insecure, but that one has to be careful in evaluating its security parameters.
\end{abstract}

\section{Introduction}

Let $u_{0}, u_{1}, \ldots$ be a linear recurrence sequence of order $n$ over the field $\mathbb{Z}_{2}$ of two elements; see [12-Chapter 8]. We may also consider each $u_{j}$ as an integer, namely 0 or 1 , and multiply by it an element $z$ of an arbitrary ring $R$, so that $u_{j} z \in R$.

We consider the following subset sum generator of pseudorandom elements. Given an $n$-dimensional vector $w=\left(w_{0}, \ldots, w_{n-1}\right) \in R^{n}$, its output is the sequence

$$
v_{i}=\sum_{0 \leq j<n} u_{i+j} w_{j}, \quad \text { for } i=0,1, \ldots,
$$

of elements of $R$. A popular choice is to take $R=\mathbb{Z}_{m}$, the residue ring modulo $m \geq 2$. The choice $m=2^{k}$ with some integer $k$ is recommended, and in particular, it is natural to choose $k=n$; see [14-Section 6.3.2]. We also consider the 
case where $m=p$ is prime. We call $\left(u_{j}\right)$ the control sequence and $w_{0}, \ldots, w_{n-1}$ the weights.

This generator, which is also known as the knapsack generator, was introduced in [19] and studied in [17, see also [14-Section 6.3.2] and [18-Section 3.7.9]. The generation algorithm is multiplication-free and involves only Boolean operations, integer additions and one modular reduction; in the case $R=\mathbb{Z}_{2^{k}}$, the reduction modulo $m=2^{k}$ is essentially for free in the binary representation. Thus it presents a very attractive alternative to pseudorandom number generators based on Boolean functions. On the other hand, its close relation to the subset sum problem could make it cryptographically strong and suitable for using in stream ciphers.

For cryptographic applications, it is usually recommended to use a linear recurrence sequence of maximal period $2^{n}-1$, however here we consider more general settings.

The linear complexity and distribution of this generator have been studied in 6, 17, 18] and have turned out to be rather attractive. Furthermore, [14 page 220] states that no weaknesses of this generator have been reported in the literature. This paper presents some weaknesses. We do not, however, consider them as lethal.

We study predictability properties of the subset sum generator and show that its security is smaller than has been assumed previously, but presumably still large enough, with appropriate parameters. In the simplest cases our attacks are based on linear algebra. In more practical settings we use lattice algorithms, namely algorithms for the short vector problem which essentially go back to the seminal paper of Lenstra, Lenstra and Lovász [11]. Thus our results add one more example to the substantial list of cryptographic constructions which have been successfully attacked by such algorithms, see [13, 15, 16].

We note that our results resemble those about predictability of various recursive pseudorandom number generators; see [2, 3, 4, 5, 8, 9, 10, and references therein.

In general, if $R=\mathbb{Z}_{m}$, the whole generator is defined by about $n(2+\log m)$ bits, where $\log z$ denotes the binary logarithm of $z>0$. Indeed, one needs $n$ bits to describe the characteristic polynomial of the control linear recurrence sequence $\left(u_{j}\right), n$ bits for its initial values, and about $n \log m$ bits to describe the weight vector $w$. Thus a brute force search through the space of all possible parameters takes about $(4 m)^{n}$ steps.

In our attacks we use polynomial time and assume that some partial information about the generator is known. However, one might as well "guess" this information; in this formulation our attacks lead to a substantial reduction of the cost of brute force search. In the same vein, some of our results deal with the generator before truncation, but one may simply "guess" the truncated parts and then apply our attacks. For example, as we have mentioned, it is suggested to discard about $\log n$ bits of each output $v_{i}$, see [14-Section 6.3.2]. Usually our attacks need only $O(n)$ consecutive outputs, so that the total number of guesses for the discarded bits is $2^{O(n \log n)}$ which, for the typically recommended values 
of $m$ near $2^{n}$, is substantially smaller than $(4 m)^{n} \asymp 2^{n^{2}}$. On the other hand, in some cases our attacks, empowered by lattice basis reduction algorithms, apply to truncated outputs directly.

The upshot is that when $n$ is large enough and both controls and weights are kept secret, we still consider the generator to be secure. A simple observation is that $2 n$ outputs give away the linear recurrence of the control bits if $m$ is even. This leads to an exhaustive search with cost $2^{n}$, and for odd $m$, we can mount an exhaustive search attack with cost $2^{2 n}$. Thus it is not clear in how far larger values of $m$ make the generator much more secure than $m=3$. (Our short vector attack becomes more expensive with growing $m$, but only by a polynomial factor.)

\section{Attacks with Known Control Sequence}

We first consider the case when the linear recurrence sequence $\left(u_{j}\right)$ is known. It is equivalent to know the characteristic polynomial and $n$ initial values, or just $2 n$ initial values; the characteristic polynomial can then be computed by the Berlekamp-Massey algorithm (see, for example, [7-Section 12.3]).

\subsection{Exact Outputs}

It is useful to express (1) in terms of the power series

$$
h_{u}=\sum_{i \geq 0} u_{i} x^{i}, \quad h_{v}=\sum_{i \geq 0} v_{i} x^{i}, \quad h_{w}=\sum_{0 \leq i<n} w_{n-i-1} x^{i}
$$

in $R[[x]]$. We show that the power series $h_{u} \cdot h_{w}$ and $x^{n-1} h_{v}$ agree at all but the small-order coefficients.

Lemma 1. Let $r=h_{u} \cdot h_{w} \operatorname{rem} x^{n-1}$ be the remainder of $h_{u} \cdot h_{w}$ on division by $x^{n-1}$. Then

$$
h_{u} \cdot h_{w}-r=x^{n-1} h_{v}
$$

Proof. We have

$$
\begin{aligned}
x^{n-1} h_{v} & =\sum_{i \geq 0} v_{i} x^{i+n-1}=\sum_{i \geq 0} \sum_{\substack{0 \leq j<n\\
}} u_{i+j} w_{j} x^{i+n-1} \\
& =\sum_{\substack{i \geq 0 \\
0 \leq j<n}} u_{i+j} x^{i+j} \cdot w_{j} x^{n-j-1}=\sum_{\substack{k+l \geq n-1 \\
0 \leq l<n}} u_{k} x^{k} \cdot w_{n-l-1} x^{l} .
\end{aligned}
$$

The bijective correspondence

$$
(i, j)=(k+l-n+1, n-l-1) \leftrightarrow(k, l)=(i+j, n-j-1)
$$

is responsible for the last equation. The condition $i \geq 0$ means that $k+l \geq n-1$. Thus the coefficient of the terms of degree at least $n-1$ in the products $x^{n-1} h_{v}$ and $h_{u} \cdot h_{w}$ coincide. 
When we take the weights as unknowns, the equations (11), or, equivalently, (2) yield a Hankel system of linear equations with the matrix

$$
H=\left(u_{i+j}\right)_{0 \leq i, j<n} .
$$

In a finite prime field, the Hankel matrix (3) is not guaranteed to be nonsingular. Our attack works by building up matrices of maximal rank from lines of the Hankel matrix (3). Accordingly, we may have to use $n$ arbitrary outputs, not necessarily the first ones. More precisely, we consider algorithms that for $i=0,1, \ldots$ either output $v_{i}$ or query $v_{i}$. The following result shows that we can do with few queries.

Theorem 2. Over a finite field $R=\mathbb{F}_{q}$ of $q$ elements, given a control sequence $\left(u_{j}\right)$ of order $n$, there is a deterministic algorithm to compute the sequence $v_{i}$ for $i=0,1, \ldots$, in polynomial time per element, making no more than $n$ queries in total.

Proof. The algorithm builds up $l \times n$ matrices $U_{l}$ consisting of rows

$$
r_{i}=\left(u_{i}, u_{i+1}, \ldots, u_{i+n-1}\right) \in \mathbb{F}_{q}^{n}
$$

for growing values of $l$, up to $n$. The matrix $U_{l}$ has rank $l$ over $\mathbb{F}_{q}$. We also store the values $v_{i}$ for the rows $r_{i}$ that appear in $U_{l}$.

We start with $U_{0}=I_{0}=\emptyset$, and consider $i=0,1, \ldots$ If $r_{i}$ is not linearly dependent over $\mathbb{F}_{q}$ on the rows of the current $U_{l}$ (this is the case in the first step, where $i=0$, unless $\left.r_{0}=0\right)$, then we set $I_{l+1}=I_{l} \cup\{i\}$ and add the row $r_{i}$ to $U_{l}$ to obtain $U_{l+1}$, of rank $l+1$. We also query and store $v_{i}$.

Otherwise we can write

$$
r_{i}=\sum_{k \in I_{l}} c_{k} r_{k}
$$

as a linear combination of the rows $r_{k}$ of $U_{l}$, with $k \in I_{l}$ and coefficients $c_{k} \in \mathbb{F}_{q}$. Then we output

$$
\begin{aligned}
\sum_{k \in I_{l}} c_{k} v_{k} & =\sum_{k \in I_{l}} c_{k} \sum_{0 \leq j<n} u_{k+j} w_{j} \\
& =\sum_{0 \leq j<n} w_{j} \sum_{k \in I_{l}} c_{k} u_{k+j} \sum_{0 \leq j<n} w_{j} u_{i+j}=v_{i} .
\end{aligned}
$$

We have to make at most $n$ queries for values $v_{i}$, since once we have $n$ linearly independent (over $\mathbb{F}_{q}$ ) rows $r_{i}$, then we can actually compute the weight vector $w$, and predict correctly ever after.

In characteristic 2, the Hankel matrix (3) is guaranteed to be nonsingular, and the algorithm simplifies as follows.

Corollary 3. Given an integer $k \geq 1$, a control sequence $\left(u_{j}\right)$ of order $n$ over $\mathbb{Z}_{2}$, and $n$ consecutive outputs $v_{i}$ for $0 \leq i<n$ over $R=\mathbb{Z}_{2^{k}}$, one can find the unknown weight vector $w \in R^{n}$ in deterministic polynomial time. 
Proof. Because $\left(u_{j}\right)$ is of order $n$ in $\mathbb{F}_{2}$, the integer Hankel matrix (3) is nonsingular modulo 2, see [12-Section 8.6], and hence also modulo $2^{k}$.

The algorithm also works over rings $R=\mathbb{Z}_{m}$ with squarefree $m \geq 2$, by using a "lazy" variant of Gaussian elimination. Here, whenever an element is to be inverted, one calculates its greatest common divisor with the current moduli (which initially is just $m$ ). If the greatest common divisor is nontrivial, one obtains a factorization of the modulus, and continues with the factors separately as new moduli. In fact, one should use the finest factorization of the moduli which is easy to calculate from these partial factorizations (see [1]).

\section{$2.2 \quad$ Truncated Outputs}

We now consider the case where a certain number $\ell$ of low-order bits of each value $v_{i}$ gets discarded before the rest is output, that is, only the "truncated" value $\left\lfloor v_{i} / 2^{\ell}\right\rfloor$ is known. Intuitively, it is clear that if the weights $w_{0}, \ldots, w_{n-1}$ are known up to the $2^{s}$ least significant bits, with $2^{s}=o\left(2^{l} / n\right)$, then these approximate values can be used to produce a sequence which with high probability equals the truncated output of the original generator.

Following this intuition, we now use algorithms for the short vector problem in lattices to find the truncations $\widetilde{w}_{j}=\left\lfloor w_{j} / 2^{s}\right\rfloor$ for $0 \leq j<n$. Although our method also works in other situations, we make various simplifying assumptions about the parameters $m, n, \ell$, and $s$. They always include the case when $\log m \sim n$, which is of greatest practical interest.

Given $k$ consecutive values $\left\lfloor v_{i} / 2^{\ell}\right\rfloor$ for $0 \leq i<k$, we define a lattice $\mathcal{L}_{k}$ as the set of all integer solutions $x=\left(x_{-1}, x_{0}, x_{1}, \ldots x_{n-1}, y_{0}, \ldots, y_{k-1}\right) \in \mathbb{Z}^{k+n+1}$ of the system of congruences

$$
x_{-1}+y_{i} 2^{\ell}\left\lfloor v_{i} / 2^{\ell}\right\rfloor x_{-1}+y_{i}-\sum_{0 \leq j<n} 2^{s} u_{i+j} x_{j} \equiv 0 \bmod m \quad \text { for } 0 \leq i<k .
$$

We use the celebrated algorithm of Lenstra, Lenstra and Lovász 11 for computing short vectors in lattices. This has become a central tool in cryptography; see [13, 15, 16] for outlines of recent progress in this area since the original result. Standard heuristic arguments, as in [16-Section 3.4], imply that the discriminant $D_{k}$ of $\mathcal{L}_{k}$ is likely to be $m^{k}$. Furthermore, the standard heuristics suggest that if a vector $\widetilde{w} \in \mathcal{L}_{k}$ is such that $\|\widetilde{w}\|$ is substantially smaller than $D_{k}^{1 /(k+n)}$, then any vector in $\mathcal{L}_{k}$ of length substantially smaller than $D_{k}^{1 /(k+n)}$ is likely to be proportional to $\widetilde{w}$.

We observe that $\mathcal{L}_{k}$ contains a very short vector

$$
z=\left(1, \widetilde{w}_{0}, \ldots, \widetilde{w}_{n-1}, z_{0}, \ldots, z_{k-1}\right),
$$

where

$$
z_{i}=v_{i}-2^{\ell}\left\lfloor v_{i} / 2^{\ell}\right\rfloor+\sum_{0 \leq j<n} u_{i+j}\left(2^{s}\left\lfloor w_{j} / 2^{s}\right\rfloor-w_{j}\right) \quad \text { for } 0 \leq i<k .
$$


We may assume that our parameters satisfy $n 2^{s} \leq 2^{\ell}$; otherwise the approximate weights cannot be used. Under this condition we have

$$
\left|z_{i}\right| \leq n 2^{s}+2^{\ell} \leq 2^{\ell+1} \quad \text { for } 0 \leq i<k .
$$

To simplify our calculations we also assume that

$$
k^{1 / 2} 2^{2 \ell+1} \leq m \quad \text { and } \quad 2^{s} \geq n+1 ;
$$

otherwise we either discard too many bits (and the generator is inefficient) or try to use a lattice of very high dimension (and the attack is infeasible). In this case,

$$
k 2^{2 \ell+2} \leq m^{2} 2^{-2 \ell} \leq m^{2} 2^{-2 s} \quad \text { and } \quad(n+2)^{1 / 2} 2^{-s}<n^{-1 / 2} .
$$

Then the Euclidean norm of the vector $z$ satisfies

$$
\|z\| \leq\left(1+n m^{2} 2^{-2 s}+k 2^{2 \ell+2}\right)^{1 / 2} \leq(n+2)^{1 / 2} m 2^{-s}<m n^{-1 / 2} .
$$

Now if we assume that $\log m \sim n$, then for $k=n^{2}-n$ we deduce

$$
D_{k}^{1 /(k+n)}=m^{k /(k+n)}=m^{1-1 / n},
$$

which is much larger than $\|z\| \leq m n^{-1 / 2}$ as long as, say, $\log m=O(n)$. Hence a nonzero multiple of $z$ is likely to be recovered by a short vector problem algorithm. Because the first component of $z$ is known, this allows us to find $z$.

\section{$3 \quad$ Attacks with Known Weights}

We now consider the dual question, where the linear recurrence sequence $\left(u_{n}\right)$ is unknown but the vector of weights $w=\left(w_{0}, \ldots, w_{n-1}\right) \in \mathbb{Z}_{m}^{n}$ is given. When we are given only a single output of the generator, then this is a subset sum problem and NP-complete. However, having several consecutive outputs allows us to mount efficient linear algebra attacks.

\subsection{Exact Outputs}

We start with even characteristic and present our results in the case when the characteristic polynomial of the control linear recurrence sequence $\left(u_{j}\right)$ is irreducible, which includes the most interesting cases of such sequences. In the general case one can obtain similar results, which however hold only for almost all weights rather than for all $w \in \mathbb{Z}_{m}^{n}$.

Theorem 4. Given an integer $k \geq 1$, the weights $w=\left(w_{0}, \ldots, w_{n-1}\right) \in R^{n}$ over $R=\mathbb{Z}_{m}$ with even $m$, and $2 n$ consecutive outputs $v_{i}$ for $0 \leq i<2 n$, not all even, one can find the controls $u=\left(u_{0}, \ldots, u_{2 n-1}\right) \in \mathbb{Z}_{2}^{2 n}$ in deterministic polynomial time, provided that the (unknown) characteristic polynomial of degree $n$ over $\mathbb{Z}_{2}$ of the control linear recurrence sequence $\left(u_{j}\right)$ is irreducible. 
Proof. The reduction of the sequence $\left(v_{i}\right)$ modulo 2 satisfies the same linear recurrent relation as the control sequence $\left(u_{j}\right)$. By assumption, this reduction is not identical to zero modulo 2. We use the Berlekamp-Massey algorithm, see [7-Chapter 7] or [12-Section 8.6], to recover the characteristic polynomial

$$
f=\sum_{0 \leq i \leq n} f_{i} x^{i} \in \mathbb{Z}_{2}[x]
$$

of this sequence, so that

$$
\sum_{0 \leq i \leq n} f_{i} u_{k+i}=0
$$

for all $k \geq 0$. The first $n$ equations in (2) plus the $n-1$ equations for the control values $u_{n}, \ldots, u_{2 n-2}$ lead to the following system of $2 n-1$ linear equations in the $2 n-1$ unknowns $u_{0}, \ldots, u_{2 n-2}$ :

$$
\left(\begin{array}{ccccccc}
w_{0} & w_{1} & \cdots & w_{n-1} & 0 & \cdots & 0 \\
0 & w_{0} & \cdots & w_{n-2} & w_{n-1} & \cdots & 0 \\
\vdots & \ddots & \ddots & \ddots & \ddots & \cdots & \vdots \\
0 & \cdots & \cdots & w_{0} & w_{1} & \cdots & w_{n-1} \\
f_{0} & f_{1} & \cdots & f_{n-1} & f_{n} & \cdots & 0 \\
\vdots & \ddots & \ddots & \ddots & \ddots & \ddots & \vdots \\
0 & \cdots & f_{0} & f_{1} & f_{2} & \cdots & f_{n}
\end{array}\right)\left(\begin{array}{c}
u_{0} \\
u_{1} \\
\vdots \\
u_{n-1} \\
u_{n} \\
u_{n+1} \\
\vdots \\
u_{2 n-2}
\end{array}\right)=\left(\begin{array}{c}
v_{0} \\
v_{1} \\
\vdots \\
v_{n-1} \\
0 \\
0 \\
\vdots \\
0
\end{array}\right) .
$$

We denote the matrix of the above system of equations by $A \in R^{(2 n-1) \times(2 n-1)}$, and observe that $A$ is the (transpose of the) Sylvester matrix of the two polynomials $f$ and

$$
w=\sum_{0 \leq i<n} w_{i} x^{i} \in \mathbb{Z}_{2}[x] .
$$

The outputs are not all even, and hence also the weights, and thus $w$ is nonzero of degree less than $n$. Since $f$ is irreducible of degree $n$, we have $\operatorname{gcd}(w, f)=1$ and hence $A$ is nonsingular. Thus we can solve the system for $u_{0}, \ldots, u_{2 n-2}$.

When the characteristic polynomial $f$ is not irreducible, the characteristic polynomial $g$ of $v_{0}, v_{1}, \ldots$ is a divisor of $f$. If the weights are chosen at random, we expect $g=f$ to hold with high probability; see [7-Section 12.4]. Furthermore, for random $w$ the condition $\operatorname{gcd}(w, f)=1$ (so that $A$ is nonsingular) holds with probability

$$
\Phi(f) / 2^{n}=\prod_{j=1}^{s}\left(1-2^{-d_{j}}\right),
$$

where $d_{1}, \ldots, d_{s}$ are the degrees of the distinct irreducible factors of $f$. Thus, $\Phi(f)$ is the polynomial analogue of Euler's $\varphi$ function. Using the fact that the number of irreducible polynomials of degree $d$ over $\mathbb{Z}_{2}[x]$ is $2^{d} / d+O\left(2^{d / 2}\right)$, one can show that this probability is also reasonably large. 
We now consider the case of an arbitrary modulus $m$. Given $k$ consecutive values $v_{i}$ for $0 \leq i<k$, we may define the lattice $\mathcal{L}_{k}$ as the set of all integer solutions $x=\left(x_{-1}, x_{0}, x_{1}, \ldots x_{k+n-2}\right) \in \mathbb{Z}^{k+n}$ of the system of congruences

$$
\sum_{0 \leq j<n} x_{i+j} w_{j}+v_{i} x_{-1} \equiv 0 \bmod m \quad \text { for } 0 \leq i<k .
$$

By (11), it contains a very short vector $\mathbf{u}=\left(-1, u_{0}, \ldots, u_{k+n-2}\right)$ with Euclidean norm at most $\|\mathbf{u}\| \leq(k+n)^{1 / 2}$. Standard heuristic arguments, as in 16 Section 3.4], imply that the discriminant $D_{k}$ of $\mathcal{L}_{k}$ is likely to be $m^{k}$.

On the other hand, also standard heuristic arguments suggest that if $\|\mathbf{u}\|$ is substantially smaller than $D_{k}^{1 /(k+n)}$, then any nonzero vector $x \in \mathcal{L}_{k}$ of length substantially smaller than $D_{k}^{1 /(k+n)}$ is likely to be proportional to $\mathbf{u}$. Thus applying any of the algorithms for the shortest vector problem, we can hope to recover $x$.

If $k \geq n+1$, then the vector $x$ gives us the values $u_{j}$ for $0 \leq j<2 n$. By the Berlekamp-Massey algorithm, one can find the characteristic polynomial of the linear recurrence sequence $\left(u_{j}\right)$ over $\mathbb{Z}_{2}$ and thus continue to generate the sequence $\left(v_{i}\right)$.

Furthermore, with $k=n+1$ we expect

$$
D_{k}^{1 /(k+n)} \sim m^{k /(k+n)} \geq m^{1 / 2}
$$

which is much larger than $(k+n)^{1 / 2}=(2 n+1)^{1 / 2}$ for all practically interesting situations.

\section{$3.2 \quad$ Truncated Outputs}

We now consider the case when some bits of the output are discarded before exhibiting the remaining bits. Although our approach works in more general settings, here consider only the case which is outlined in [14-Section 6.5.6]. In this case $t=2^{n}-1, m=2^{n}$ and $\ell=\lceil\log n\rceil$ bits of each value $v_{i}$ get discarded before the rest is output, that is, only the "truncated" values $\left\lfloor v_{i} / 2^{\ell}\right\rfloor$ are known.

Given $k$ consecutive values $\left\lfloor v_{i} / 2^{\ell}\right\rfloor$ for $0 \leq i<k$, we define a lattice $\mathcal{L}_{k}$ as the set of all integer solutions $x=\left(x_{-1}, x_{0}, x_{1}, \ldots x_{k+n-2}, y_{0}, \ldots, y_{k-1}\right) \in \mathbb{Z}^{2 k+n}$ of the system of congruences

$$
\sum_{0 \leq j<n} x_{i+j} w_{j}+2^{\ell}\left\lfloor v_{i} / 2^{\ell}\right\rfloor x_{-1}+y_{i} \equiv 0 \bmod m \quad \text { for } 0 \leq i<k .
$$

Again we observe that the discriminant of $\mathcal{L}_{k}$ is likely to be $D_{k}=m^{k}$. We also see that it contains a very short vector

$$
z=\left(-1, u_{0}, \ldots, u_{k+n}, z_{0}, \ldots, z_{k-1}\right),
$$

where $z_{i}=2^{\ell}\left\lfloor v_{i} / 2^{\ell}\right\rfloor-v_{i}$ for $0 \leq i<k$, whose Euclidean norm satisfies

$$
\|z\| \leq\left(k+n+k\left(2^{\ell}-1\right)\right)^{1 / 2}=\left(k 2^{\ell}+n\right)^{1 / 2} \leq(2 k n+n)^{1 / 2} .
$$


We see that if $k=\lceil\log n\rceil$, then $\|z\| \leq(2 n \log n+O(n))^{1 / 2}$, while

$$
D_{k}^{1 /(2 k+n)}=2^{k n /(2 k+n)} \geq n^{n /(2 k+n)}=n^{1+o(1)}, \quad \text { for } n \rightarrow \infty,
$$

is much larger. Certainly increasing the value of $k$ increases the chances that $z$ is much shorter than any other non-parallel vectors in $\mathcal{L}_{k}$ and thus can be found by an appropriate algorithm for the shortest vector problem.

We have conducted several tests for values of $n$ up to $n=100$ with $m$ a 100 bit prime. In each case, the short vector computed provided correctly the control sequence. In all cases, there have not been other "smallish" short vectors in the lattice. These experiments confirm that the algorithm always finds the control sequence, at least for sufficiently large problems of cryptographically interesting sizes.

\section{Final Remarks}

As noted before, our results do not rule out the possibility of successfully using the subset sum generator for cryptographic purposes. They merely imply that the security is less than its naive estimate based on counting unknown bits in the parameters defining the generator. Thus with a careful choice of these parameters this generator might turn out to be very useful and reliable.

It would be very interesting to obtain rigorous proofs for the heuristic attacks described in this paper. Besides being of theoretic value, this may also give further insight on the structure and thus security of the subset sum generator.

For convenience, we have assumed that $R$ is a ring, so that we could use the language of the power series ring $R[[x]]$. But the construction of the generator applies to any semigroup $R$. Now if, for example, $R$ is a finite cyclic group of order $m$ and the output sequence is given "as is", without discarding any information, then the prediction problem over $R$ can be reduced to a prediction problem over $\mathbb{Z}_{m}$ by computing the discrete logarithms of $v_{0}, v_{1}, \ldots$. At the small key sizes for which we already expect security, the discrete logarithm problem is not hard. Similar arguments apply also to groups which are not necessarily cyclic. However, this reduction to discrete logarithms does not work if some information about each generated value $v_{i}$ is discarded before the generator outputs the rest of $v_{i}$.

For example, one can use this idea in one of the cryptographically most interesting groups, namely the group of rational points of an elliptic curve over a finite field $\mathbb{F}_{p}$, where $p$ is a prime. We may choose the size $m \approx p \approx 2^{n}$ of the group sufficiently large, and discard the $\log n$ low-order bits of the $x$-coordinate of the point before using it as pseudorandom output. Then none of our attacks works, and at the current state of knowledge the only available attack on this generator is the brute force search over all parameters defining this generator. Certainly this construction deserves a further study.

With our attacks, an exhaustive search to break a subset sum generator requires about $2^{u}$ many values, where $u$ is the following "effective key size", and $m \approx 2^{n}:$ 


\begin{tabular}{c|c} 
group & key size $u$ \\
\hline $\mathbb{Z}_{m}, m$ even & $n$ \\
$\mathbb{Z}_{m}, m$ odd & $2 n$ \\
elliptic curve, truncated output & $n^{2}$
\end{tabular}

We conclude with an observation which may seem somewhat paradoxical. While we believe that truncation of the output sequence is a good idea, it must be applied with great care. Indeed, although truncation provides less information to the attacker, he only has to solve the simpler task of finding suitable approximations to the weights. This is exactly the observation that underlies our attack in Section 2.2. This attack does not work if the number of truncated bits $\ell$ is too small. On the other hand, if this value is too large, the attack becomes prohibitively expensive. It is an interesting question to estimate the "hardest" value of $\ell$ (as a function of $m$ and $n$ ), which takes into account the cost of algorithms for the short vector problem, both theoretic and heuristic.

\section{References}

1. E. Bach, J. Driscoll and J. Shallit, 'Factor refinement', J. Algorithms, 15 (1993), 199-222.

2. S. R. Blackburn, D. Gomez-Perez, J. Gutierrez and I. E. Shparlinski, 'Predicting the inversive generator', Lect. Notes in Comp. Sci., Springer-Verlag, Berlin, 2898 (2003), 264-275.

3. S. R. Blackburn, D. Gomez-Perez, J. Gutierrez and I. E. Shparlinski, 'Predicting nonlinear pseudorandom number generators', Math. Comp., (to appear).

4. S. R. Blackburn, D. Gomez-Perez, J. Gutierrez and I. E. Shparlinski, 'Reconstructing noisy polynomial evaluation in residue rings', J. Algorithms, (to appear).

5. E. F. Brickell and A. M. Odlyzko, 'Cryptoanalysis: A survey of recent results', Contemp. Cryptology, IEEE Press, NY, 1992, 501-540.

6. A. Conflitti and I. E. Shparlinski, 'On the multidimensional distribution of the subset sum generator of pseudorandom numbers', Math. Comp., 73 (2004), 10051011.

7. J. von zur Gathen and J. Gerhard, Modern computer algebra, Cambridge University Press, Cambridge, 2003.

8. A. Joux and J. Stern, 'Lattice reduction: A toolbox for the cryptanalyst', J. Cryptology, 11 (1998), 161-185.

9. H. Krawczyk, 'How to predict congruential generators', J. Algorithms, 13 (1992), $527-545$.

10. J. C. Lagarias, 'Pseudorandom number generators in cryptography and number theory', Proc. Symp. in Appl. Math., Amer. Math. Soc., Providence, RI, 42 (1990), $115-143$.

11. A. K. Lenstra, H. W. Lenstra and L. Lovász, 'Factoring polynomials with rational coefficients', Mathematische Annalen, 261 (1982), 515-534.

12. R. Lidl and H. Niederreiter, Finite fields, Cambridge University Press, Cambridge, 1997.

13. D. Micciancio and S. Goldwasser, Complexity of lattice problems, Kluwer Acad. Publ., 2002. 
14. A. J. Menezes, P. C. van Oorschot and S. A. Vanstone, Handbook of applied cryptography, CRC Press, Boca Raton, FL, 1996.

15. P. Q. Nguyen and J. Stern, 'Lattice reduction in cryptology: An update', Lect. Notes in Comp. Sci., Springer-Verlag, Berlin, 1838 (2000), 85-112.

16. P. Q. Nguyen and J. Stern, 'The two faces of lattices in cryptology', Lect. Notes in Comp. Sci., Springer-Verlag, Berlin, 2146 (2001), 146-180.

17. R. A. Rueppel, Analysis and design of stream ciphers, Springer-Verlag, Berlin, 1986.

18. R. A. Rueppel, 'Stream ciphers', Contemporary cryptology: The science of information integrity, IEEE Press, NY, 1992, 65-134.

19. R. A. Rueppel and J. L. Massey, 'Knapsack as a nonlinear function', IEEE Intern. Symp. of Inform. Theory, IEEE Press, NY, 1985, 46. 